\title{
Assessment of Irritable Bowel Syndrome Using Traditional Chinese Medicine - A Critique of Recent Studies
}

\author{
Jennifer Brett* \\ University of Bridgeport, USA
}

Submission: February 10, 2017; Published: August 14,2017

*Corresponding author: Jennifer Brett, ND, L.Ac., Director, Univ. of Bridgeport Acupuncture Institute, Tel: 203-576-4122/ 203-767-1939;

Email: jbrett@bridgeport.edu

\section{Opinion}

Irritable bowel syndrome (IBS) is a common complaint in the US. [1] It is characterized by abdominal pain relieved by defecation and the absence of any other identifiable bowel disorders. The differential diagnosis of IBS includes celiac disease, small intestinal bacterial overgrowth [2], bile acid diarrhea $[3,4]$, exocrine pancreatic insufficiency [5], or inflammatory bowel disease. [6] It can be complicated by stress $[7,8]$, large bowel dysbiosis [9], lactose intolerance [8] and other less common food intolerances, medications, nutrients (calcium can cause constipation; magnesium and vitamin $\mathrm{C}$ can cause diarrhea) and disorders characterized by prostaglandin/ leukotriene imbalances [10]. Biomedical diagnosis of IBS is one of exclusion only. The Rome Criteria [11] notes

\section{IBS criteria}

A. Recurrent abdominal pain or discomfort at least 3 days/month in last 3 months associated with two or more of the following

B. Improvement with defecation

C. Onset associated with a change in frequency of stool

D. Onset associated with a change in form (appearance of stool)

E. Routine laboratory studies (complete blood count, chemistries) are normal in IBS. Diagnostic "red flags" such as weight loss, blood in the stool and night awakening from abdominal pain are absent.

IBS can manifest as "Diarrhea-predominant IBS" "Constipation-predominant IBS" or as a "Mixed IBS" syndrome where both constipation and diarrhea are present. [12]

No specific studies can positively prove the presence of IBS. [12]
Routine laboratory studies (complete blood count, chemistries) are normal in IBS.

"Red Flag" or "Alarm" or atypical symptoms which are not compatible with IBS [12] include:
A. Rectal bleeding
B. Nocturnal or progressive abdominal pain
C. Weight loss
D. Laboratory abnormalities such as anemia, elevated inflammatory markers, or electrolyte disturbance

In Traditional Chinese Medicine (TCM), the diagnosis of IBSlike syndromes is equally complex. Practitioners need to utilize all four exams to correctly identify both the root and branch issues that complicate bowel function. In a recent meta-analysis of acupuncture for the treatment of IBS [13], the authors found no significant evidence that acupuncture was effective in treating IBS. I propose that this conclusion could have been predicted by both the simplistic approach to the TCM treatments and the lack of sophistication of the TCM diagnoses of this disorder.

In most of the studies of acupuncture for IBS, the TCM "diagnosis" of IBS is characterized by Spleen qi deficiency with or without liver overacting on Spleen. [14-29] This limitation of diagnoses to those of the zang "Pi" and "Gan" ignores the panoply of other possible diagnoses for IBS symptoms. IBS may include diarrhea, constipation or both along with abdominal pain.

The TCM differential diagnosis of diarrhea/loose stools include:
A. Spleen qi xu
B. Spleen yang $\mathrm{xu}$
C. Large intestine damp heat 
D. Kidney qi or yangxu

E. Stomach Food Stagnation

F. Stomach Fire

G. Stomach Dampness

H. Stomach Cold

I. Stomach Qi Deficiency

J. Liver overacting on the Spleen

The TCM differential diagnosis of constipation includes:
A. Large Intestine qi xu
B. Large intestine jinyexu
C. Stomach qixu
D. Stomach heat [shi]
E. Stomach yin $\mathrm{xu}$
F. Kidney yin $\mathrm{xu}$
G. Liver overacting on the bowels
H. Liver overacting on Stomach
I. Blood deficiency
J. Lung qi xu

The TCM Differential of abdominal pain includes:

A. Stomach Food Stagnation

B. Cold, Damp, or Heat attacking the Stomach

C. Rebellious Stomach Qi

D. Stomach Qi Deficiency with Stagnation

E Qi and blood stagnation in the ST channel

F. Small intestine qi stagnation

G. Cold, heat or phlegm obstruction in the bowels

H. Constrained Liver qi

I. Dai Mai dysfunction

J Stagnation in the Yin Chiao and/or Yin Wei Mai

K. Stagnation in the Chong Mai

L. Retention of cold, cold damp or damp heat in the ST Divergent channel

IBS may be any combination of the above diagnoses. Treatment of these combination of problems can be quite complex. Treatment of IBS with acupuncture (alone or with electro acupuncture, moxibustion, cupping, etc.) is similarly complex. The recent "placebo-blinded" studies of acupuncture for IBS limit the treatment arm to six or fewer prescribed acupuncture points. $[14,15,18,29-31]$ The most commonly used points from these studies include LI 4 (Hegu), BL 60 (Kunlun) (as a placebo point), LR 3 (Taichong), ST 36 (Zusanli), SP 6 (Sanyinjiao), Ren 12 (Zhiongwan), ST 21 (Liangmen), ST 25 (Tianshu), HT 7 (Shenmen), Du 20 (Baihui), ST 37(Shangjuxu) With such simplified diagnostic and treatment parameters, it is not surprising that the meta-analysis does not match experienced practitioners' clinical experiences when applying personalized, individualized treatments of IBS. It is also possible that some of those enrolled in the studies did not, in fact, have IBS.

The TCM approach to IBS must include a thorough medical diagnosis to exclude other causes of bowel dysfunction, as well as a thorough TCM exam to identify both root and stem patterns that are affecting the functioning of the stomach, spleen, liver and small and large bowels. The treatment arms need to take into account the TCM pattern differentiation in the use of needles with or without electro acupuncture and moxibustion for the various patterns identified. While such study design is complex, it would better mirror the treatments being offered IBS patients in the US.

\section{References}

1. Drossman DA, Camilleri M, Mayer EA, Whitehead WE (2008) AGA technical review on irritable bowel syndrome. Gastroenterology 123(6): 2108-2131.

2. Sandler RS, Everhart JE, Donowitz M (2002) The burden of selected digestive diseases in the United States. Gastroenterology 122(5): 15001511.

3. Longstreth GF, Thompson WG, Chey WDFunctional (2006) bowel disorders. Gastroenterology 130(5): 1480-1491.

4. Swarbrick ET, Hegarty JE, Bat L (1980) Site of pain from the irritable bowel. Lancet 2(8192): 443-446.

5. Manning AP, Thompson WG, Heaton KW, Morris AF (1978) Towards positive diagnosis of the irritable bowel. Br Med J 2(6138): 653-654.

6. Lovell RM, Ford AC (2012) Prevalence of gastro-esophageal refluxtype symptoms in individuals with irritable bowel syndrome in the community: a meta-analysis. Am J Gastroenterol 107(12): 1793-1801.

7. Fukudo S (2013) Stress and visceral pain: Focusing on irritable bowel syndrome. Pain 154(Suppl 1): S63-S70.

8. Yan J, Fox M, Cong Y, Chu H, Zheng X, et al. (2014) Lactose intolerance in irritable bowel syndrome patients with diarrhoea: the roles of anxiety, activation of the innate mucosal immune system and visceral sensitivity. Aliment Pharmacol Ther 39(3): 302-311.

9. Duboc H, Rainteau D, Rajca S, Humbert L, Farabos D, et al. (2012) Increase in fecal primary bile acids anddysbiosis in patients with diarrhea-predominant irritable bowel syndrome. Neurogastroenterol Motil 24(6): 513-547.

10. Balestra B, Vicini R, Cremon C, Zecchi L,DothelG (2012) Colonic mucosal mediators from patients with irritable bowel syndrome excite enteric cholinergic motor neurons. Neurogastroenterol Motil 24(12): 1118-1570.

11. Ford AC, Bercik P, Morgan DG, Bolino C, Pintos-Sanchez MI, et al. (2013) Validation of the Rome III Criteria for the Diagnosis of Irritable Bowel Syndrome in Secondary Care. Gastroenterology 145(6): 1262-1270. 
12. Wald AMD, Talley NJ (2016) Clinical manifestations and diagnosis of irritable bowel syndrome in adults.

13. Manheimer E, Wieland LS, Cheng K (2012) Acupuncture for irritable bowel syndrome: systematic review and meta-analysis. Am J Gastroenterol 107(6): 835-847.

14. Macpherson H, Bland M, Bloor K (2010) Acupuncture for irritable bowel syndrome: a protocol for a pragmatic randomised controlled trial. BMC Gastroenterology 10: 63.

15. Fireman Z, Segal A, Kopelman Y (2001) Acupuncture treatment for irritable bowel syndrome. A double-blind controlled study. Digestion 64(2): 100-103

16. Liu M (1995) Clinical report of ear acupuncture treatment for functional gastric disorder. Shanghai J Acupunct Moxibust 14: 247-248.

17. Ruepert L, Quartero AO, De Wit NJ (2011) Bulking agents, antispasmodics and antidepressants for the treatment of irritable bowel syndrome. Cochrane Database Syst Rev 10(8):

18. Schneider A, Enck P, Streitberger K (2006) Acupuncture treatment in irritable bowel syndrome. Gut 55(5): 649-654

19. Sun JH, Wu XL, Xia C (2011) Clinical evaluation of soothing gan and invigorating pi acupuncture treatment on diarrhea-predominant irritable bowel syndrome. Chin J Integr Med 17(10): 780-785.

20. Xiong X, Lin Y (2008) Acupuncture plus traditional Chinese herb medicine in treating 42 patients with diarrhea-predominant IBS. Journal of Fujian University of TCM 18: 38-40.

21. Xue Y, Tian X (2009) The clinical research of the relationship between radicals and irritable bowel syndrome and the effect of acupuncture. Chinese Archives of Traditional Chinese Medicine 27: 111-112.

22. Zeng Y, Bao Y, Chu J (2010) Effect of moxibustion for diarrhea-dominant irritable bowel syndrome patients in dog days. Chinese Archives of Traditional Chinese Medicine 28: 1774-1776.
23. Li W (2009) Treatment of warming needle moxibustion combined with spinal manipulation on irritable bowel syndrome. China Pract Med 4: 212-213.

24. Liu GZ (1997) Treatment of acupuncture and moxibustion plus psychotherapy for irritable bowel syndrome. Chinese Acupunct Moxibust 10: 611-612.

25. Liu Q Wang Z, Zhang W (2010) Clinical observation on geshanxiaoyao decoction combining acupuncture effect on the life quality of patients with irritable bowel syndrome. Journal Of Zhejiang Chinese Medical University 34: 510-513.

26. Lowe C, Depew W, Vanner S (2000) A placebo-controlled, double-blind trial of acupuncture in the treatment of irritable bowel syndrome (IBS). Gastroenterology 118(1): A3168.

27. Reynolds JA, Bland JM, Macpherson H (2008) Acupuncture for irritable bowel syndrome an exploratory randomised controlled trial. Acupunct Med 26(1): 8-16.

28. An G, Li N, Zhai G (2010) Evaluation of the therapeutic effect of acupuncture and moxibustion on irritable bowel syndrome. Shanghai J Acu-mox 29: 354-356.

29. Anastasi JK, Mcmahon DJ, Kim GH (2009) Symptom management for irritable bowel syndrome: a pilot randomized controlled trial of acupuncture/moxibustion. Gastroenterol Nurs 32(4): 243-255.

30. Shi X, Luo J, Tan T (2010) Clinical observation of electroacupuncture on diarrhea-predominant irritable bowel syndrome. J New Chinese Med 42: 72-74.

31. Lembo AJ, Conboy L, Kelley JM (2009) A treatment trial of acupuncture in IBS patients. Am J Gastroenterol 104(6): 1489-1497.
This work is licensed under Creative Commons Attribution 4.0 Licens DOI: 10.19080/JCMAH.2017.03.555608 